\title{
Europe et communication : le poids des résistances culturelles dans la construction européenne
}

Jean Michel Djian

\section{(2) OpenEdition}

1 Journals

Édition électronique

URL : http://journals.openedition.org/communicationorganisation/2333

DOI : 10.4000/communicationorganisation.2333

ISSN : $1775-3546$

Éditeur

Presses universitaires de Bordeaux

Édition imprimée

Date de publication : 1 mai 2000

ISSN : 1168-5549

Référence électronique

Jean Michel Djian, «Europe et communication : le poids des résistances culturelles dans la

construction européenne », Communication et organisation [En ligne], 17 | 2000, mis en ligne le 27 mars 2012, consulté le 01 mai 2019. URL : http://journals.openedition.org/

communicationorganisation/2333 ; DOI : 10.4000/communicationorganisation.2333

Ce document a été généré automatiquement le 1 mai 2019.

(c) Presses universitaires de Bordeaux 


\title{
Europe et communication : le poids des résistances culturelles dans la construction européenne
}

\author{
Jean Michel Djian
}

1 L'usage massif des technologies d'information et de communication pour conquérir plus rapidement des parts de marché en Europe se heurte à des résistances d'ordre culturel que les hommes politiques et les économistes n'imaginaient probablement pas. Même si à l'échelle de l'Histoire, l'Europe se fait vite (moins d'un demi-siècle entre le traité de Rome et l'entrée en vigueur de l'euro), la gestion volontariste mise en œuvre pour faire de l'intégration européenne un enjeu politique majeur, continue de se conjuguer difficilement avec les habitudes et les modes de vie des habitants du Vieux Continent. Estce la difficulté de transcrire un message européen qui soit autre chose qu'une série d'avantages matériels et économiques, ou l'incapacité d'une classe politico-médiatique à théoriser la question? Toujours est-il que le discours européen des années 1980 et $a$ fortiori celui des années 1990, poursuit son déphasage avec les populations des pays d'Europe. Il n'y aurait, sinon, aucune raison de voir une partie significative de l'opinion se détourner d'un processus qu'elle vit comme une fatalité et non comme un rêve. Il apparaît en effet que nous sommes probablement arrivés au bout de cette logique politico-médiatique dans la représentation du mythe moderne de l'Europe, celle qui a permis de confondre allégrement construction européenne avec progrès scientifique et démocratie avec individualisme. Et c'est probablement dans l'absence d'un idéal collectif intellectuellement construit qu'il faut chercher l'essoufflement dont l'Europe est aujourd'hui la victime. Trois raisons peuvent expliquer l'enchaînement des confusions et ce retour inquiétant à un nationalisme rampant.

\section{Déficit de sens}

2 La première d'entre elles tient moins à la masse d'outils disponibles pour informer ou s'informer sur ce qu'est l'Europe qu'au déficit de sens communiqué par ces supports. En 
décrétant l'Europe dans les années 1960, les classes politiques des pays membres ont tenté une sorte de pari sur la modernité, relayés par des médias très conformistes sur la question. L'ensemble de la population s'est longtemps contentée d'enregistrer la mise en œuvre d'un processus qui, globalement, ne suscitait aucun débat. Au début des années 1970, seuls les communistes refusaient officiellement l'Europe. Une forme d'évidence naturelle, fondée sur une logique géographique, puis culturelle, reposant sur l'acceptation philosophique du droit à la différence a permis aux Européens de se sentir en communauté c'est-à-dire ensemble. Le marché a fait le reste. Synonyme de progrès, il a subtilement permis à l'individualisme naissant de se mouvoir dans une sorte de démocratie économique affranchie des archaïsmes idéologiques qui sévissaient jusqu'alors. Il a, grâce aux technologies de l'information, (télévision, informatique) réduit les distances symboliques, et a permis, du fait d'une libéralisation des transports et des barrières douanières, de baisser considérablement le coût desdits transports et donc de mieux circuler. Ce même marché a tenté dans la foulée, une homogénéisation de la consommation (volonté de mettre en place des campagnes publicitaires ciblées simultanément dans plusieurs pays) quitte à ce que chaque pays renonce à des particularismes culturels considérés comme trop « folkloriques ».

Cette façon de voir nie les ressorts anthropologiques qui structurent la dimension contemporaine de la civilisation européenne. Au moment où l'Europe naissait, une classe intellectuelle nationale structurée à droite autour de Raymond Aron et à gauche autour de Jean-Paul Sartre, s'est désintéressée de ce mouvement de fond politique, pour laisser quelques hommes d'État pourtant courageux (Monnet, Schuman) s'aventurer seuls. L'Europe est le produit de ce contexte dont la caractéristique principale s'apparente à du simplisme politique. De Rougemont était et reste inconnu et il a fallu qu'Edgar Morin publie en France à la fin des années 1980 Penser l'Europe et Hans Magnus Enzensberger en Allemagne, Europe, Europe !pour que les intellectuels se remettent à penser et la classe dirigeante à réfléchir. Seul le franco-allemand continuait d'agiter les esprits sous la férule des Grosser ou Rovan. C'est, en gros, ce qui s'est fait pendant une trentaine d'années jusqu'au Traité de Maastricht, date à laquelle une certaine conception de l'Europe a été freinée par un mouvement de fond hostile à toute standardisation. Personne jusqu'alors ne s'était aperçu que «les petites gens » refusaient ou pouvaient refuser l'Europe par incompréhension de l'enjeu. C'est pourtant ce qui s'est passé. Une césure s'est alors opérée entre d'un côté, ceux qui savaient et, de l'autre, ceux qui ne savaient pas.

\section{L'absence d'un message fédérateur}

4 La deuxième raison est le corollaire de la première. Il ne peut exister un message unique destiné à 300 millions de personnes d'origines géographiques, culturelles et linguistiques si diverses. Ce qui est possible à l'échelle d'un pays (pour prouver la nécessité d'une réforme par exemple) ne l'est pas sur un conglomérat de nations qui cherchent à s'unir. Malgré le degré de militance important constaté depuis bien longtemps à l'échelle de chacune d'entre elles (le Mouvement européen existe depuis le congrès de La Haye en 1947), une part importante des populations ose refuser ouvertement l'Europe parce qu'elle est synonyme de marché, de bureaucratie et de Cheval de Troie de la mondialisation. Il existe, en quelque sorte, un déphasage historique entre d'une part une classe politique éduquée dans un environnement idéologiquement marqué par la Guerre froide mais qui n'a pas pensé un instant que la Chute du Mur de Berlin était une 
hypothèse dans la construction de l'Histoire et l'opinion qui, à cause de son détachement politique, pouvait se l'imaginait. Il en a résulté une variété de "micro-paradigmes » naissants, fruits de la diversité mais hostiles à toute rationalisation d'un nouveau modèle européen que le politique tente aujourd'hui encore d'installer.

\section{Des moyens de communication inadaptés}

5 La troisième raison enfin se rapporte au décalage qui a existé entre d'une part la démocratisation des moyens de communication (apparition des radios privées dans les années 1980, naissance des chaînes thématiques dans les années 1990, numérisation tous azimut et généralisation d'Internet et des portables) et d'autre part le besoin d'Europe. Lorsque Monnet et quelques-uns ont décrété en 1947 que la paix sur le Continent passait par la construction d'une Europe forte et unie, les gouvernements d'alors ne disposaient que de moyens de communication de type propagandiste c'est-à-dire à sens unique Les voyages étaient chers et réservés à une élite et la pratique des langues étrangères n'était pas considérée, surtout en France, comme valorisante. Il en a résulté une forme d'incompréhension entre une classe politique plutôt moderne qui n'avait de cessé de démonter les bienfaits d'une communauté du Charbon et de l'Acier et une génération du «baby boom » pour qui l'Europe, faute de moyens pour la visiter et d'instruments pour la comprendre, se résumait à un vague projet politique porté par des conservateurs plutôt éclairés.

\section{Une classe politique trop peu européenne}

6 Si les jeunes d'alors avaient disposé de moyens pour communiquer à bas prix, nul doute que l'Europe politique eût été pensée autrement. Cette inadéquation dans le temps explique en partie ce déficit d'image et le refus d'une certaine catégorie de population d'en saisir les vertus. Mais il est saisissant en même temps d'observer que l'offre politique d'Europe a toujours été en déphasage avec une demande culturelle d'Europe. Comme si les gouvernements ne s'étaient jamais rendu compte que les stratégies impressionnantes de communication politique sur la question européenne ne pouvait s'affranchir d'une expérience pratique concomitante. Or que constate-t-on? Les décideurs de la vie publique nationale sont très peu européens. Ils méconnaissent beaucoup trop les institutions, à Bruxelles ou à Strasbourg. Les partis politiques, français en particulier, continuent d'envoyer au Parlement «les seconds couteaux » et l'administration ne sait souvent pas faire la distinction entre une directive et un règlement communautaire, ce qui signifie a fortiori une incapacité à gérer les flux d'informations en provenance de Bruxelles et une incapacité à peser sur les décisions. La technique du lobbyisme étant très peu prisée par les Français, les organisations publiques ou privées se contentent le plus souvent d'opérer de simples relations publiques là où il faudrait véritablement défendre de dossiers en usant des rapports de forces stratégiques et politiques. Ce que d'autres peuples comme la Grèce ou l'Italie, pourtant latins, ont appris depuis qu'ils ont intégré l'Union.

On peut aujourd'hui en France et en toute légitimité, tenir un discours cohérent sur la construction européenne tout en perpétuant une pratique nationale des nouvelles 
réalités qui s'imposent du fait de cette Construction. Et sans que pour autant ces acteurs publics ne tranchent la contradiction.

8 Cette résistance culturelle à l'Europe se niche en France là où elle est le moins attendue : au cœur d'une administration véritablement jacobine mais qui décide et détient certains leviers de commande pour informer; dans le milieu rural, là même où les crédits communautaires sont de loin les plus importants (si ce n'est que les logiques qui soustendent les subventions aux agriculteurs sont vécues à juste titre comme incompréhensibles); dans le petit commerce enfin, qui voit dans l'Europe et la mondialisation les symboles de la déchéance des valeurs républicaines et/ou chrétiennes.

De toute cette évolution les partis politiques des années 1990 ont fait leur miel. Les clivages se sont opérés au delà de la traditionnelle opposition gauche/droite et l'Europe s'est mise à inventer des postures politiques inédites dont des événements, comme le conflit des Balkans ont permis l'émergence. Même si un point de non retour est atteint sur le fait européen, rien n'est acquis sur le plan institutionnel. L'heure de vérité approche. Poursuivre l'aventure suppose, comme le disait Edgar Morin (Penser l'Europe), de prendre conscience « d'une communauté de destin »C'est le seul débat qui compte et il arrive au moment même où les manifestations régionalistes cristallisent une aspiration des peuples à s'émanciper des tutelles centralisatrices.

\section{De nouvelles formes de communication?}

Les résistances culturelles à la construction européenne se cristallisent autour des formes nouvelles de communication. La génération des $45 / 55$ ans, celle qui détient aujourd'hui les leviers politiques n'a qu'une idée convenue et une pratique superficielle d'Internet. Or force est de constater que les nouvelles technologies de l'information ont "déterritorialisé » l'espace européen chez les 20/30 ans. Le concept de réseau est une réalité tangible. Aujourd'hui, les structures traditionnelles continuent de communiquer verticalement quand les jeunes le font horizontalement. En ce sens, l'Europe et la technologie font bon ménage mais cela profite d'abord à une génération qui n'a pas le pouvoir et n'a qu'une connaissance élémentaire de l'Europe.

Le risque est grand de voir une fracture s'opérer entre d'une part des Européens investis dans un modèle institutionnel dépassé et des nouveaux Européens pour qui le vieux continent est un territoire pouvant très bien se contenter de fonctionner en réseau. Une telle conception "basique» de l'Europe ne fera que renforcer les sentiments d'appartenance culturelle d'une troisième catégorie de population qui considère qu'il est $\mathrm{du}$ ressort du politique de donner un sens à ce combat. Cette dernière, pourtant la plus capable de faire des sacrifices, ne se contentera plus de «consommer » de l'Europe sans qu'une alternative idéologique à l'État nation lui soit proposée. Or, rien ne vient, puisque même les thèses réformistes du fédéralisme sont aujourd'hui tabou à quelques exceptions près. $11 \mathrm{y}$ a là une réaction purement démocratique, élémentaire, qui semble avoir été sous-estimée par cette accélération de l'Histoire dont les politiques et les intellectuels ont été au fond plus les spectateurs que les acteurs. Comme pour les Lumières ou la Révolution, les peuples ont besoin qu'un projet soit nourri par l'utopie, la pensée et l'action. Pour l'heure, l'Europe doit se contenter de l'action. C'est-à-dire des agissements du marché.

\section{Revues à consulter :}




\section{BIBLIOGRAPHIE}

Commune aux articles d'Anne-Marie Autissier et de Jean-Michel Djian.

AUTISSIER Anne-Marie, L'Europe culturelle en pratique, Paris, Chronique AFAA/ministère des Affaires étrangères, 1999, $147 \mathrm{p}$.

BOSSUAT Gérard, L'Europe des Français, (1943-1959), Paris, Publications de la Sorbonne, 1997,471 p. BOSSUAT Gérard, « Des lieux de mémoire pour l'Europe unie », in Vingtième Siècle, n 61, janviermars 1999, pp. 56-69.

BRAGUE Rémi, Europe, la voie romaine, Paris, Gallimard, Coll. Folio, 1999, 272 p. (première édition : Le Centurion, Paris, 1992)

BROSSAT Caroline, La culture européenne : définitions et enjeux, Bruxelles, Éditions Bruylant, 1999, $534 \mathrm{p}$.

ENZENSBERGER Hans Magnus, Europe, Europe! Paris, Gallimard, 1988, 380 p. Girault René (dir.) (Collaboration Bossuat Gérard), Les Europe des Européens, Paris, Publications de la Sorbonne, 1993, $156 \mathrm{p}$.

GIRAULT René (dir.), Identité et conscience européennes au XXe siècle, Paris, Hachette, 1994, 234 p.

KASTORYANO Riva (dir.), Quelle identité pour l'Europe ? Le multiculturalisme à l'épreuve, Paris, Presses de Sciences Po, 1998, 267 p.

MORIN Edgar, Penser l'Europe, Paris, Gallimard 1987, 262 p.

FREYMONT Jacques et Williams ANDREWS, (dir.), (Collaboration : Monnet

Gertrude), Le patrimoine européen : unité et singularité, Actes de la Table ronde organisée à Genève du 29 juin au 2 juillet 1983, Genève, Centred'études pratiques de la négociation internationale, 1984, $212 \mathrm{p}$.

ROUGEMONT (de) Denis, Lettre ouverte aux Européens, Paris, Albin Michel, 1970.

SCARDIGLI Victor, L’Europe de la diversité, Paris, Éditions du CNRS, 1993, 240 p.

Wolton Dominique, L'Europe, la dernière utopie, Paris, Flammarion, 1993, 454 p.

Wolton Dominique, « Le désert européen de la communication », in Internet et après, Une théorie critique des nouveaux médias, Paris, Flammarion, 1999, pp. 163-185.

Zuppinger Renaud (dir.), Représentations du passé, Patrimoine, musées, problématiques identitaires et culturelles en Europe, Saint-Denis, Numéro spécial de la revue Le Fil d'Ariane, Institut d'études européennes de l'Université de Paris VIII, 1997, 122 p. 
Europe, la force de la culture, Paris, Fondation Jean Jaurès, 1999, 191 p.

« Histoire des peuples européens et identité commune », in Tousignant Nathalie, (sous la direction de), Les identités de l'Europe : repères et prospective, Actes du colloque de Louvain-la-neuve, le 9 mai 1996, UCL, 1998 pp. 41-60.

L'Europe, une identité plurielle, Actes du colloque organisé par les Éditions Autrement, avec le soutien du ministère chargé des Affaires européennes, Paris, Éditions Autrement, 2000, 179 pp.

Les frontières politiques du patrimoine culturel européen, Coll. des textes de la Quatrième conférence des Ministres responsables du patrimoine culturel, Strasbourg, Conseil de l'Europe, 1996, 11 p.

«Le repli culturel de l’Europe », Dossier de la revue Esprit n² 265, juillet 2000, pp. 88-155.

Les traces du pèlerinage à Saint-Jacques de Compostelle dans la culture européenne, Strasbourg, Conseil de l'Europe, Coll. Patrimoine culturel n²0,1992, 145 p.

\section{RÉSUMÉS}

L'usage massif des technologies d'information et de communication pour conquérir plus rapidement des parts de marché en Europe se heurte à des résistances d'ordre culturel que les hommes politiques et les économistes n'imaginaient probablement pas. De telles difficultés tiennent au déficit de sens du message européen envoyé par les institutions communautaires, à l'absence d'un message fédérateur, aux préoccupations strictement nationales des dirigeants politiques et à la coïncidence entre la signature du Traité de Rome et l'existence de moyens de communication considérés comme "unilatéraux ». Les nouvelles formes de communication ont contribué à « déterritorialiser » l'Europe et à pratiquer un fonctionnement «horizontal » alors que leurs aînés en sont restés à un modèle institutionnel dépassé.

The massive use of information technologies in order to rapidly increase market shares faces cultural resistance in Europe which probably was not foreseen by politicians and economists. The obstacles come from the failure of the European message sent by the EC to become meaningful as well as the absence of any federative message. Also involved are the strictly national concerns of politicians. When the Treaty of Rome was signed, top-down communications were the only ones in existence. New forms of communication have contributed to deterritorialize the European space and set up a horizontal network whereas the EC remains attached to an obsolete institutional model.

\section{INDEX}

Mots-clés : déficit de sens, message européen, modèle institutionnel

\section{AUTEUR}

\section{JEAN MICHEL DJIAN}

Jean-Michel Djian est professeur associé à l'Université de Paris VIII. Docteur en Sciences politiques, il travaille aussi comme journaliste. Il a été directeur du Monde de l'Éducation de 1996 à 1999. Il dirige la collection « Mémoires du siècle » à la Chaîne Histoire. Il a 
publié : La politique culturelle, Paris, Le Monde/Marabout, 1997, Le triomphe de l'ordre, Paris, Flammarion, 2000. 